\title{
Collisional evolution and the resulting mass distribution of interplanetary dust
}

\author{
Hiroshi Ishimoto \\ The Graduate School of Science and Technology, Kobe University, Nada, Kobe 657-8501, Japan
}

(Received October 8, 1997; Revised March 3, 1998; Accepted March 13, 1998)

\begin{abstract}
On the basis of numerical approaches for the collisional and orbital evolution of dust particles, the number density distribution of interplanetary dust with the mass range of $m \geq 10^{-12} \mathrm{~g}$ is investigated. The slope of the mass distribution of dust particles strongly depends on the radial dependence of dust production by their parent bodies, and the collisional interaction between particles. Specifically, the $m^{-7 / 3}$ dependence of the number density distribution at $1 \mathrm{AU}$ for $m \geq 10^{-6} \mathrm{~g}$ can be explained through the balance between the collisional loss of particles and the dust supply, whereas the $m^{-4 / 3}$ dependence for $10^{-12} \mathrm{~g} \leq m \leq 10^{-6} \mathrm{~g}$ particles is derived from simple PoyntingRobertson orbital decay. A possible model of the dust populations of asteroidal, cometary, and Edgeworth-Kuiper belt origin that is consistent with the observed dust flux at a solar distance of $1 \mathrm{AU}$ is presented.
\end{abstract}

\section{Introduction}

The Interplanetary Meteoroids Flux (IMF) model derived by Grün et al. (1985) has been widely accepted as a standard model of interplanetary dust flux at $1 \mathrm{AU}$. One of the interesting points of the IMF and other interplanetary flux models (e.g. McDonnell, 1978; Le Sergeant and Lamy, 1980) is that the flux curve changes its slope around the particle masses of $10^{-12} \mathrm{~g}$ and $10^{-6} \mathrm{~g}$. Although some attempts have been made (e.g. Mukai, 1989), the curve of the flux of the IMF model has not been explained in detail, i.e. why does such a slope appear in the flux curve? Moreover, the spatial and number density distributions of interplanetary dust inside and outside $1 \mathrm{AU}$ are not well known.

One approach to solving the above problems is analytic estimation from ground-based observations of zodiacal cloud and in-situ dust measurements by space probes. From data obtained by ground based observations and space-probe measurements Divine (1993) designed "five populations" of interplanetary dust, mainly from the orbital analysis of particles. Since his analysis does not consider the relationship between dust particles and their origins, we cannot see any grounds for the existence of such a dust population, although his results do suggest some implications when considering the origin and evolution of interplanetary dust particles.

Dust particles in interplanetary space probably consist of dust groups of many origins. After the discovery of the influx of interstellar dust (e.g. Grün et al., 1993), a significant portion of sub-micron sized interstellar dust flux has been reported, even inside 5 AU (e.g. Baguhl et al., 1995a; Grün et al., 1995a,b; Grün et al., 1997). Moreover, recent estimation of the number of Edgeworth-Kuiper belt objects indicates that EKOs may be an important dust source, as well as asteroids and comets (e.g. Backman et al., 1995; Stern, 1996; Yamamoto and Mukai, 1998).

When particles are released from a parent body, the radial

Copy right (C) The Society of Geomagnetism and Earth, Planetary and Space Sciences (SGEPSS); The Seismological Society of Japan; The Volcanological Society of Japan; The Geodetic Society of Japan; The Japanese Society for Planetary Sciences. distribution of the particles is relatively similar to that of the parent body. The particles will arrive at $1 \mathrm{AU}$ after their orbital evolution and mutual collisions. As mentioned by Grün et al. (1985), collisional evolution will play an important role in deducing the observed nature of interplanetary dust. In this work, numerical simulations for the number density of dust particles are performed, taking into account their origin and evolution. Furthermore, we present a possible model of dust populations that is consistent with the dust flux model at $1 \mathrm{AU}$.

\section{Numerical Methods}

If we assume that the number density $n(m, r) d m d r$ of dust particles for arbitrary mass $m$ at a solar distance $r$ is stable in time, the radial dependence of $n(m, r)$ can be expressed as (e.g. Ishimoto, 1998)

$$
\frac{\partial n(m, r)}{\partial r}=-\frac{n(m, r)}{r}\left(2+\frac{r}{\left|v_{r}\right|} \frac{\partial\left|v_{r}\right|}{\partial r}\right)+\frac{d t}{d r} \frac{d n^{\prime}(m, r)}{d t},
$$

where $v_{r}$ is the mean radial velocity of the particles. The first term of the right hand side of Eq. (1) comes from geometric concentration due to the radial change of the particles' location. The time variation in number density without the geometric concentration, i.e. the second term of the right hand side of Eq. (1), comes therefore from dust production and loss $\left(d n^{\prime} / d t\right.$ is the time variation in number density due to dust production and loss). In this work, dust production by comets and asteroids and collisional loss of particles are assumed. To avoid some complexities, we take into account only the variation of number densities for $m \geq 10^{-12} \mathrm{~g}$ particles; particles smaller than $10^{-12} \mathrm{~g}$ therefore only act as the loss term for larger particles by collisions. Specifically,

$$
\frac{d n^{\prime}(m, r)}{d t}=\frac{d n_{\mathrm{ag}}}{d t}+\frac{d n_{\mathrm{cg}}}{d t}-\frac{d n_{\mathrm{pp}}}{d t}-\frac{d n_{\mathrm{beta}}}{d t}-\frac{d n_{\mathrm{is}}}{d t}
$$

where $d n_{\mathrm{ag}} / d t$ and $d n_{\mathrm{cg}} / d t$ are the dust supplied by asteroids and by comets. $n_{\mathrm{pp}} / d t, n_{\text {beta }} / d t$, and $n_{\text {is }} / d t$ are, respectively, 


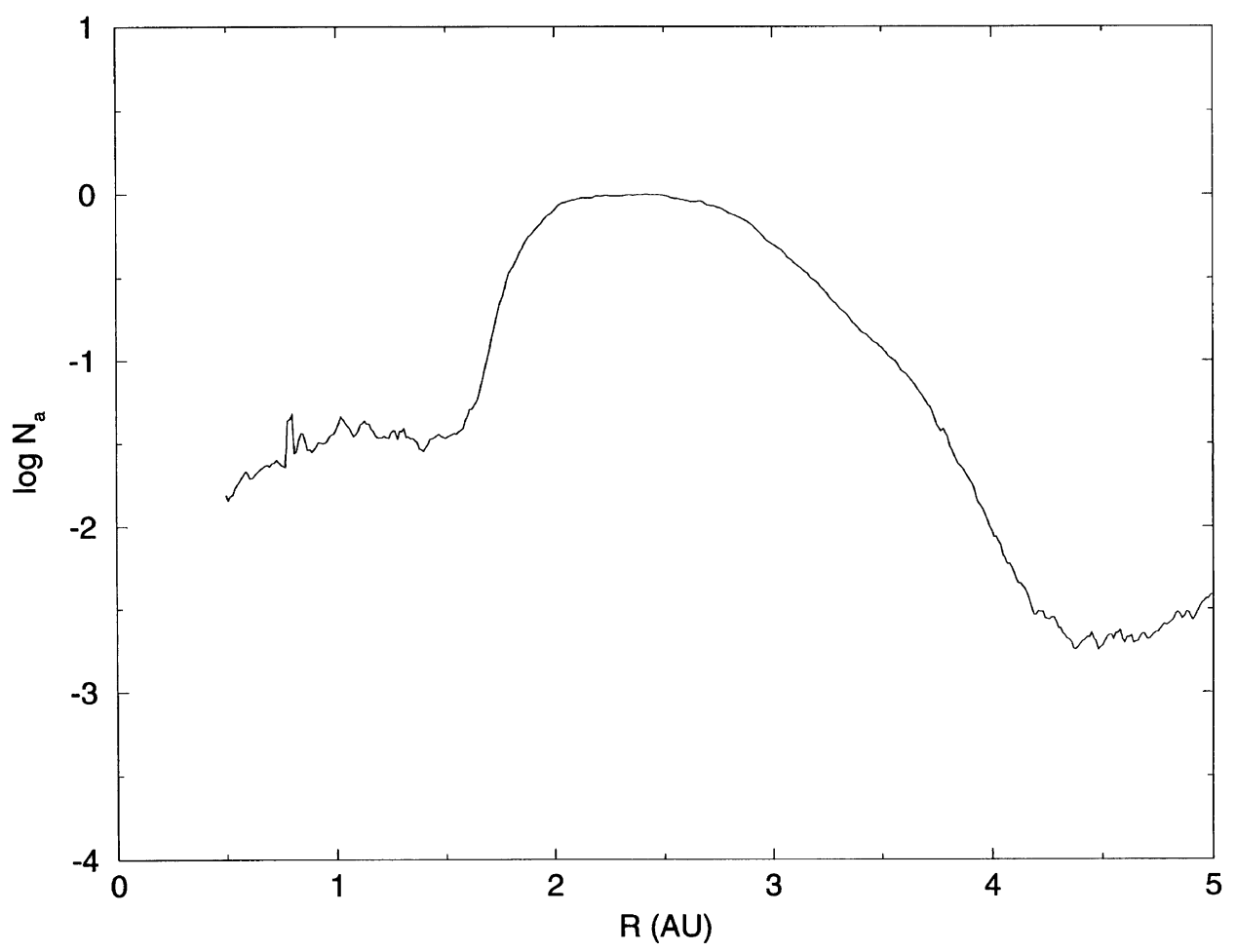

Fig. 1(a). A relative number density $N_{a}$ of asteroids as a function of solar distances $R$. The orbital data of "The Asteroid Orbital Elements Database" published by Lowell Observatory are used to estimate $N_{a}$.

loss terms by collisions between $m \geq 10^{-12} \mathrm{~g}$ particles, by collisions with $\beta$-meteoroids and with interstellar particles.

For simplicity, we consider the mean radial velocity $v_{r}$ as that given by the Poynting-Robertson effect. In this case,

$$
v_{r}=\frac{d r}{d t} \sim-\frac{2 \beta G M_{\odot}}{r c},
$$

where $\beta$ is the ratio of the solar radiation pressure to the solar gravity on the grain. $G, M_{\odot}$, and $c$ are, respectively, the gravitational constant, mass of the sun and speed of light. Using Eq. (3), Eq. (1) can be written as

$$
\frac{\partial n(m, r)}{\partial r} \sim-\frac{n(m, r)}{r}-\frac{r c}{2 \beta G M_{\odot}} \frac{d n^{\prime}(m, r)}{d t} .
$$

This simple approach may not be appropriate for particles with high orbital eccentricities. Indeed, the value $v_{r}$ for particles with highly eccentric orbits has to be estimated by combining the mean radial velocity due to the PoyntingRobertson effect with that due to the eccentric orbit. Therefore, Eqs. (3) and (4) are only rough approximations for the radial dependence of the number densities of particles for particles with eccentric orbits. Nevertheless, the effects of eccentricity are applied to the radial dependence of dust production by parent bodies described below.

The mechanism of dust production differs between asteroids and comets. Asteroidal particles will be produced mainly by impacts between asteroids. The production rate of asteroidal particles is, therefore, proportional to the collision rate of asteroids. Assuming the number density of asteroids $N_{a}$ at a solar distance $r$, the dust production becomes

$$
\frac{d n_{\mathrm{ag}}}{d t} \propto N_{a}^{2} v_{i} g_{a}(m) .
$$

$v_{i}$ is the impact velocity between asteroids, and $g_{a}(m)$ is the mass distribution function of impact ejecta. For the first order approximation, we neglect the other possible contributions, i.e. asteroidal impact ejecta by collisions with comets and interplanetary/interstellar meteoroids. These contributions may become important if the number densities of such projectiles are larger than $N_{a}$ and effective dust production occurs.

On the other hand, cometary particles are ejected with vaporized volatile material. Therefore, the dust production rate will be proportional to the comet's activity. For a simple estimate, we assume that all the cometary particles are produced at the perihelion distance of the comet. Then the orbit averaged dust production rate is, approximately, proportional to the inverse of the orbital period of the comet. Summing up, for the $j$-th comet which crosses the distance $r$,

$$
\frac{d n_{\mathrm{cg}}}{d t} \propto \sum_{j} \frac{g_{c}(m)}{q_{j}^{2}} \cdot \frac{1}{T_{j}} \cdot \frac{1}{r^{2} v_{r j} T_{j}}
$$

where $q_{j}, T_{j}$, and $v_{r j}$ are, respectively, the perihelion distance of the $j$-th comet, its orbital period, and its radial velocity at $r . g_{c}(m)$ is the mass distribution of ejected particles. Note that the last term of Eq. (6) denotes the relative probability of the existence of ejected particles at a distance $r$ (e.g. Kessler, 1981).

The relative values of $N_{a}$ for arbitrary solar distance $r$ are derived by using "The Asteroid Orbital Elements Database" published by the Lowell Observatory, and also the relative values of $d n_{\mathrm{cg}} / d t$ are deduced from the orbits of known short period comets (Figs. 1(a) and 1(b)). 


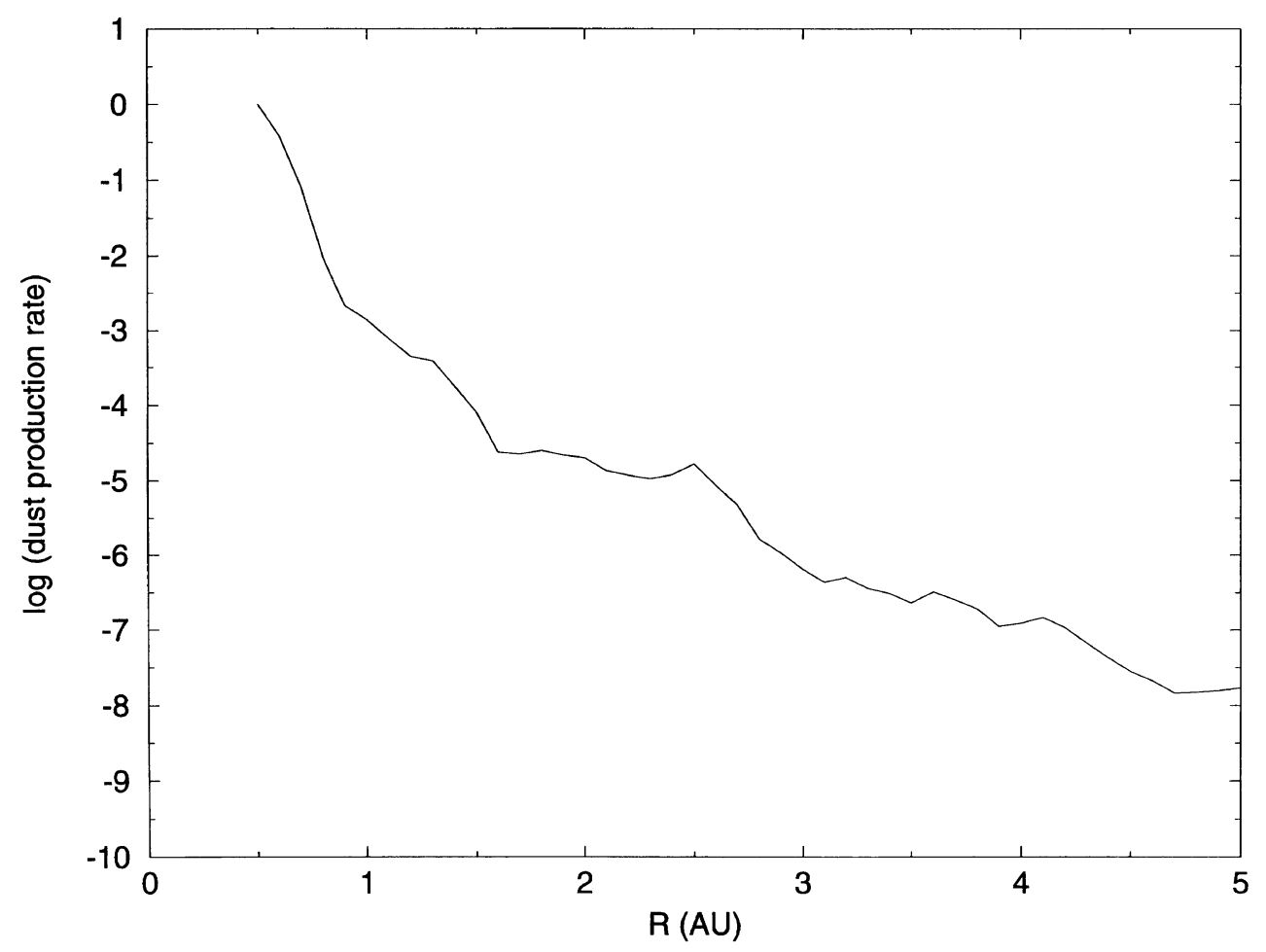

Fig. 1(b). A relative dust production rate $d n_{\mathrm{cg}} / d t$ under a simple assumption, i.e. dust production occurs only at a perihelion of parent comet, derived from the orbital distribution of known short period comets.

The terms of collisional loss in Eq. (2) are, respectively,

$$
\begin{aligned}
\frac{d n_{\mathrm{pp}}}{d t}= & n(m, r) v_{\mathrm{pp}} \int_{m_{\text {min }}}^{m_{\max }} \sigma_{\mathrm{pp}} n(m, r) d m, \\
\frac{d n_{\text {beta }}}{d t}= & n(m, r) v_{\text {beta }} \\
& \cdot \int_{m_{\text {bmin }}}^{m_{\text {bmax }}} \sigma_{\text {beta }} n\left(m, r_{0}\right)\left(\frac{r}{r_{0}}\right)^{-1.5} d m, \\
\frac{d n_{\text {is }}}{d t}= & n(m, r) \frac{\sqrt{v_{\mathrm{k}}^{2}+v_{\text {is }}^{2}}}{v_{\text {is }}} \sigma_{\text {is }} f_{\text {is }} C,
\end{aligned}
$$

where $\sigma_{\mathrm{pp}}, \sigma_{\text {beta }}$, and $\sigma_{\text {is }}$ are collisional cross sections of the particle of interest with $m \geq 10^{-12} \mathrm{~g}$ particles, with $\beta$ meteoroids, and with interstellar particles. The mean impact velocity of $m \geq 10^{-12} \mathrm{~g}$ particles $v_{\mathrm{pp}}$ is $(2 / 3) v_{\mathrm{k}}$, where $v_{\mathrm{k}}$ is the Keplerian velocity at a distance $r$ (e.g. Grün et al., 1985). For the impact velocity of $\beta$-meteoroids $v_{\text {beta }}, \sqrt{2} v_{\mathrm{k}}$ is used, assuming parabolic orbits for $\beta$-meteoroids that are generated deep inside 1 AU. A mass range $0.5 \times 10^{-15} \mathrm{~g} \leq$ $m<10^{-12} \mathrm{~g}$ for $\beta$-meteoroids is assumed from the condition $\beta \geq 0.5$ for a spherical "astronomical silicate" (Draine and Lee, 1984). It should be noted that the production of small fragments by particle-particle collisions is neglected for simplicity, and therefore Eq. (8) is not self-consistent if we assume that the $\beta$-meteoroids are produced by particleparticle collisions. However, since the number density of interplanetary dust is higher inside $1 \mathrm{AU}$ than outside, it is not so unrealistic to assume that most $\beta$-meteoroids are formed inside $1 \mathrm{AU}$.

For the mean flux of interstellar particles $f_{\text {is }}$ and the mean velocity $v_{\text {is }}$, the values observed by Ulysses in the vicinity of Jupiter $\left(f_{\text {is }}=8 \times 10^{-5} \mathrm{~m}^{-2} \mathrm{sec}^{-1}, v_{\text {is }}=26 \mathrm{~km} \mathrm{sec}^{-1}\right.$ (Grün et al., 1993)) are used. Although recent observational results by Galileo and Ulysses strongly suggest a significant contribution of interstellar particles, at least outside 1.8 AU (e.g. Baguhl et al., 1995b; Grün et al., 1997), interstellar flux as a function of solar distance has not been well investigated. Therefore, a parameter $C$ is adopted as the penetration coefficient of interstellar particles inside $5 \mathrm{AU}(0 \leq C \leq 1)$.

For the mass distribution functions in Eqs. (5) and (6), a simple power law distribution $g_{a}(m), g_{c}(m) \propto m^{-5 / 3}$ was applied, referring to laboratory measurements of impact debris by Nakamura and Fujiwara (1991) and to in-situ measurements of impact dust at comet $\mathrm{P} /$ Halley by Mazets et al. (1986).

In this work, we try to estimate from the shape of their mass distribution the contribution of asteroidal/cometary particles in interplanetary space. The absolute value of the total mass of asteroidal/cometary particles will not be examined directly. However, we use the condition that the number densities for asteroidal/cometary particles are comparable with those of the IMF model at $1 \mathrm{AU}$ (Grün et al., 1985) with particle masses of $10^{-12} \mathrm{~g} \leq m \leq 10^{-9} \mathrm{~g}$ (e.g. Dermott et al., 1994a; Jayaraman and Dermott, 1996).

\section{Results and Discussion \\ 3.1 Preliminary results}

Preliminary results for the case of a single dust source are shown in Figs. 2 and 3. Using iterative calculations, the number densities of asteroidal particles (Fig. 2) and cometary particles (Fig. 3) at $1 \mathrm{AU}$ are chosen to be comparable with those of the IMF model in their mass range $10^{-12} \mathrm{~g} \leq m \leq 10^{-9} \mathrm{~g}$. 


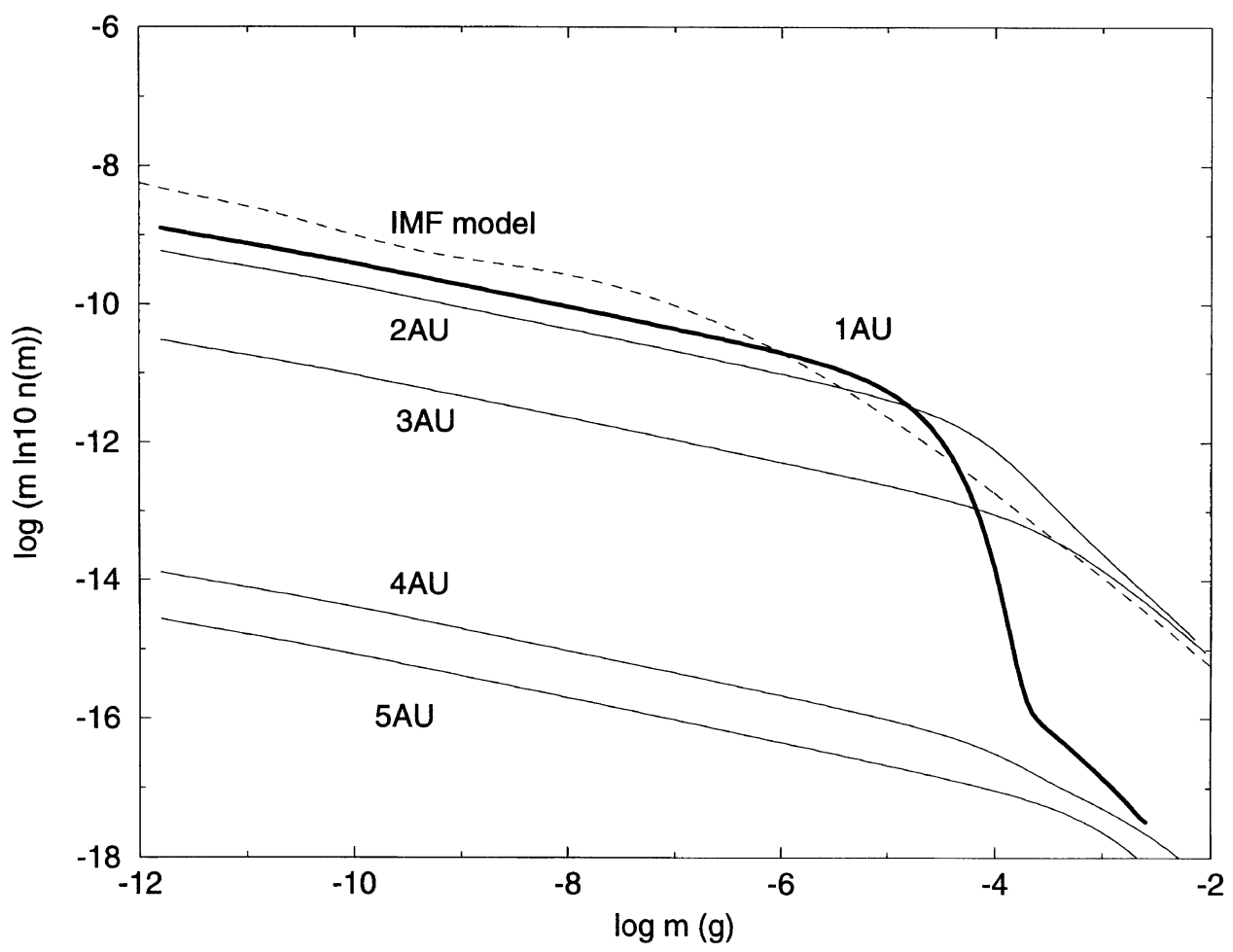

Fig. 2. Preliminary results of the orbital and collisional evolution for the mass distribution of dust particles in the case of single asteroidal population. The number density distribution of interplanetary dust particles is plotted at a different solar distance. Dashed line demotes the IMF model at a solar distance of $1 \mathrm{AU}$. For the loss terms of number densities, the mutual collisions of particles with masses $m \geq 10^{-12} \mathrm{~g}$ and the collisions with $\beta$-meteoroids are taken into account. The resulting distribution in Fig. 1(a) is applied for the radial dependence of the production rate of particles from asteroids. The absolute value of asteroidal ejecta is set to become a comparable value of the number density of particles with $10^{-12} \mathrm{~g} \leq m \leq 10^{-9} \mathrm{~g}$ in those observed for IMF model. See text for detailed explanations.

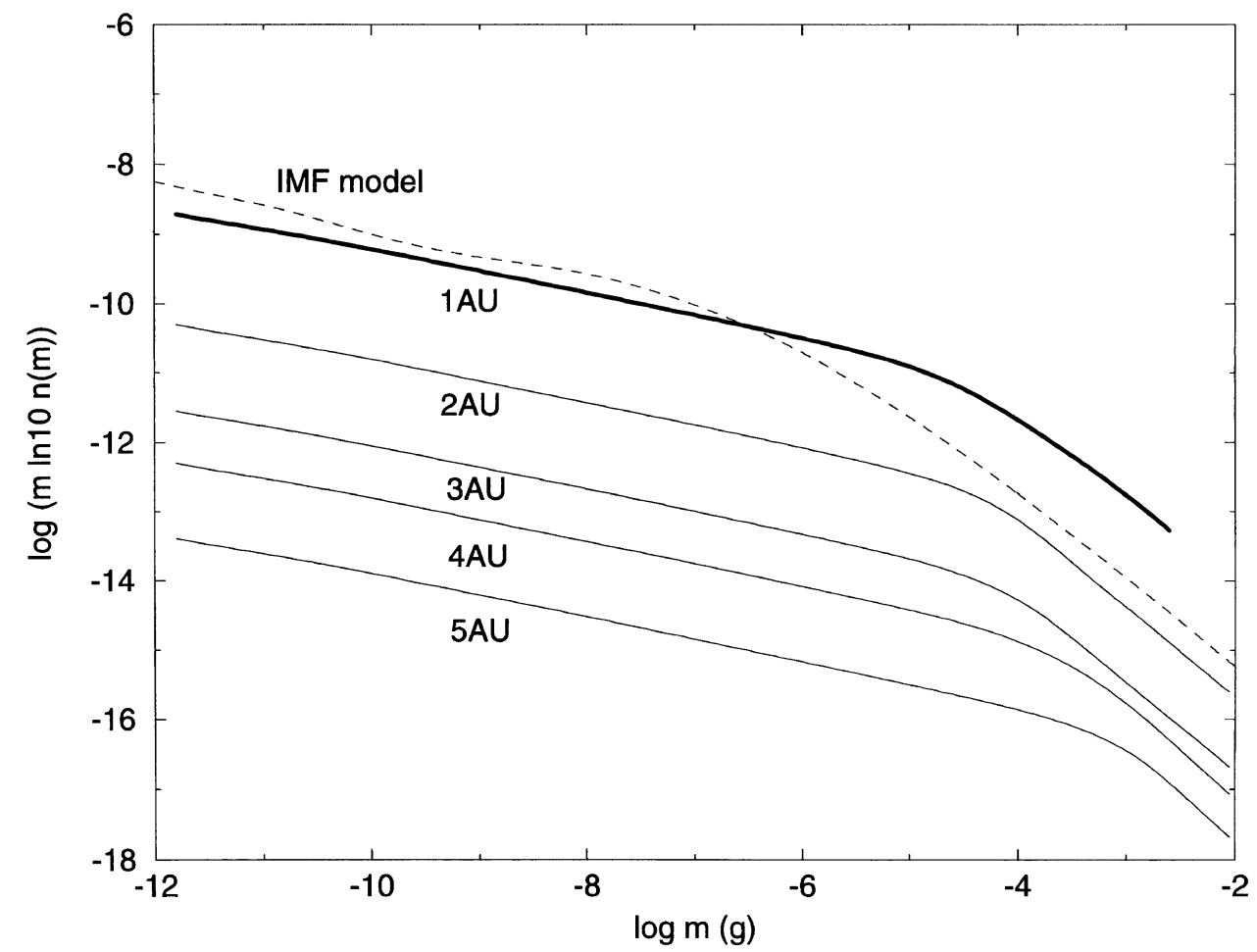

Fig. 3. The same as Fig. 2, but in the case of single cometary population. 
Both calculations assume no interstellar flux inside $5 \mathrm{AU}$ $(C=0)$. If particles have not been affected by collisional destruction, the curve of their number densities keeps the $n(m) \propto m^{-4 / 3}$ dependence, because of the radial velocity of Poynting-Robertson orbital decay. Specifically,

$$
n(m) \propto g(m) \cdot \frac{1}{\beta} \sim m^{-5 / 3} \cdot m^{1 / 3}=m^{-4 / 3} .
$$

This slope is similar to that of the IMF model in the mass range of $10^{-12} \mathrm{~g} \leq m \leq 10^{-7} \mathrm{~g}$. If the above estimate is adequate, we can say that the interplanetary dust particles with $10^{-12} \mathrm{~g} \leq m \leq 10^{-7} \mathrm{~g}$ at 1 AU have not been affected by particle collisions, because of the fast orbital decay by Poynting-Robertson drag compared with the collision lifetime of particles. Furthermore, this slope of the number density distribution has no relation to whether the particles were generated near $1 \mathrm{AU}$ or generated far from $1 \mathrm{AU}$. Therefore, this slope of the number density distribution provides no information about the radial distribution of dust sources. An important point of the IMF number density distribution is that the slope of $m^{-4 / 3}$ changes and becomes steeper for $m \geq 10^{-7} \mathrm{~g}$. As the particles' mass increases, the radial velocity of Poynting-Robertson decay decreases. Thus the timescale of Poynting-Robertson decay exceeds the timescale of the mutual collisions of particles falling toward the sun. If particles are generated far beyond $1 \mathrm{AU}$ and collisional destruction becomes effective, then the number density distribution at $1 \mathrm{AU}$ will show a rapid decrease beyond the critical mass $m_{c}$, because almost all of the particles with $m \geq m_{c}$ will be destroyed by mutual collisions before arriving at $1 \mathrm{AU}$.

In the case of asteroidal particles (Fig. 2), we have found from our model calculations that a rapid decrease in their number density occurs beyond $m \sim 10^{-5} \mathrm{~g}$. This is because most asteroidal particles are generated in the region of the asteroid belt (see Fig. 1(a)) and particles with $m \geq 10^{-5} \mathrm{~g}$ released in the asteroid belt have almost disappeared through particle collisions before arriving at $1 \mathrm{AU}$. However, the IMF model has no such sudden decrease in number densities. This fact leads to a fundamental difficulty in explaining the interplanetary dust flux observed at $1 \mathrm{AU}$ in a wide mass range by the dust particles of asteroidal origin alone.

In the case of cometary particles (Fig. 3), we found that the slope of the number density distribution also changes around $10^{-5} \mathrm{~g}$. However, the slope of $m \geq 10^{-5} \mathrm{~g}$ is flatter than that of asteroidal particles. This is because of the difference in the radial distributions of the production rate of dust particles by asteroids and comets. Comets' dust production increases with decreasing solar distance (see Fig. 1(b)), and a continuous supply of cometary particles in the vicinity of 1 AU therefore prevents a sudden drop in the number density distribution. The slope of $m \geq 10^{-5} \mathrm{~g}$ particles in Fig. 3 can be explained by a balance between the collisional loss and gain of dust particles in this mass range. If the main mechanism of particle loss is collisional destruction, then the loss rate in Eq. (2) is approximately proportional to $n(m) \cdot m^{2 / 3}$. Assuming a balance between collisional loss and dust supply,

$$
n(m) \propto m^{-5 / 3} \cdot m^{-2 / 3}=m^{-7 / 3} .
$$

Note that this mass dependence of the number density dis- tribution is similar to that of the IMF model for $m \geq 10^{-7} \mathrm{~g}$. This implies that the interplanetary dust particles detected at 1 AU with a mass of $m \geq 10^{-7} \mathrm{~g}$ are supplied near $1 \mathrm{AU}$ directly from their parent bodies, and are not coming from outside 1 AU by Poynting-Robertson orbital decay. Furthermore, the collisional destruction of particles is balanced with the dust supply, at least for particles in this mass range.

\subsection{Contribution of each dust group}

In the case of both Figs. 2 and 3, the number density of particles exceeds that of the IMF model in the mass range $m \sim 10^{-6} \mathrm{~g}$ when we normalize the derived values with the IMF flux around $10^{-12} \mathrm{~g} \leq m \leq 10^{-9} \mathrm{~g}$. This indicates that the collisional loss is not sufficient for the preliminary cases of Figs. 2 and 3. However, such disagreement is minor and can be solved if we take, for example, a larger value for the penetration coefficient of interstellar particles $C$. That is, the effect of collisions of interstellar particles with larger particles reduces the relative abundance of particles with $10^{-6} \mathrm{~g} \leq m \leq 10^{-4} \mathrm{~g}$. From the numerical calculations, it is found that the best fit to the IMF model is achieved when we apply the factor $C=0.1$. Although interstellar contribution inside $5 \mathrm{AU}$ has not yet been evaluated in detail, $C=0.1$ will be an acceptable value if we average over $1 \sim 5 \mathrm{AU}$ (e.g. Baguhl et al., 1995a,b).

Two possible results are shown in Figs. 4 and 5. In both calculations, the asteroidal and cometary particles are set to contribute significantly to the number density at 1 AU of particles with $10^{-12} \mathrm{~g} \leq m \leq 10^{-9} \mathrm{~g}$ (in this calculation, resultant mixing between asteroidal and cometary dust in this mass range was about $8: 5$ at $1 \mathrm{AU}$ ). To fit the calculated number density distribution at $1 \mathrm{AU}$ to that of the IMF model, it is not necessary to consider the contribution from asteroidal particles. However, the asteroidal dust group can not be neglected in explaining several types of evidence of its presence, derived from IRAS and COBE observations (e.g. Dermott et al., 1984; Dermott et al., 1994a,b; Reach et al., 1995; Jayaraman and Dermott, 1996), even if cometary dust has partially contributed to the Earth's resonant ring.

In Fig. 5, an additional group of dust particles from Edgeworth-Kuiper belt objects has been applied as a possible dust source outside 5 AU. The number density distribution of EKO particles has been estimated by using a similar calculation to that described in the previous section, assuming the radial distribution of EKOs from Backman et al. (1995) and the factor of interstellar flux $C=1.0$ outside $5 \mathrm{AU}$. Also, the number density of EKO particles in $10^{-12} \mathrm{~g} \leq m \leq 10^{-9} \mathrm{~g}$ is set to be comparable with that of the IMF model. The resulting shape of the number density distribution of EKO dust is consistent with the estimation derived by Liou et al. (1996). Namely, the group of EKO particles can contribute only in the mass range $m \leq 10^{-9} \mathrm{~g}$ (less than $10 \mu \mathrm{m}$ in size) even if it exists at $1 \mathrm{AU}$, because of collisional destruction by interstellar particles during their Poynting-Robertson decay time. It should be noted that the existence of EKO particles has no significant role in the number density distribution of particles at $1 \mathrm{AU}$ in the mass range $\geq 10^{-9} \mathrm{~g}$ (see Figs. 4 and 5).

As shown in Fig. 4, if we assume only two main dust sources, i.e. asteroids and short period comets, the number density of dust particles in $m \geq 10^{-12} \mathrm{~g}$ will decrease by 


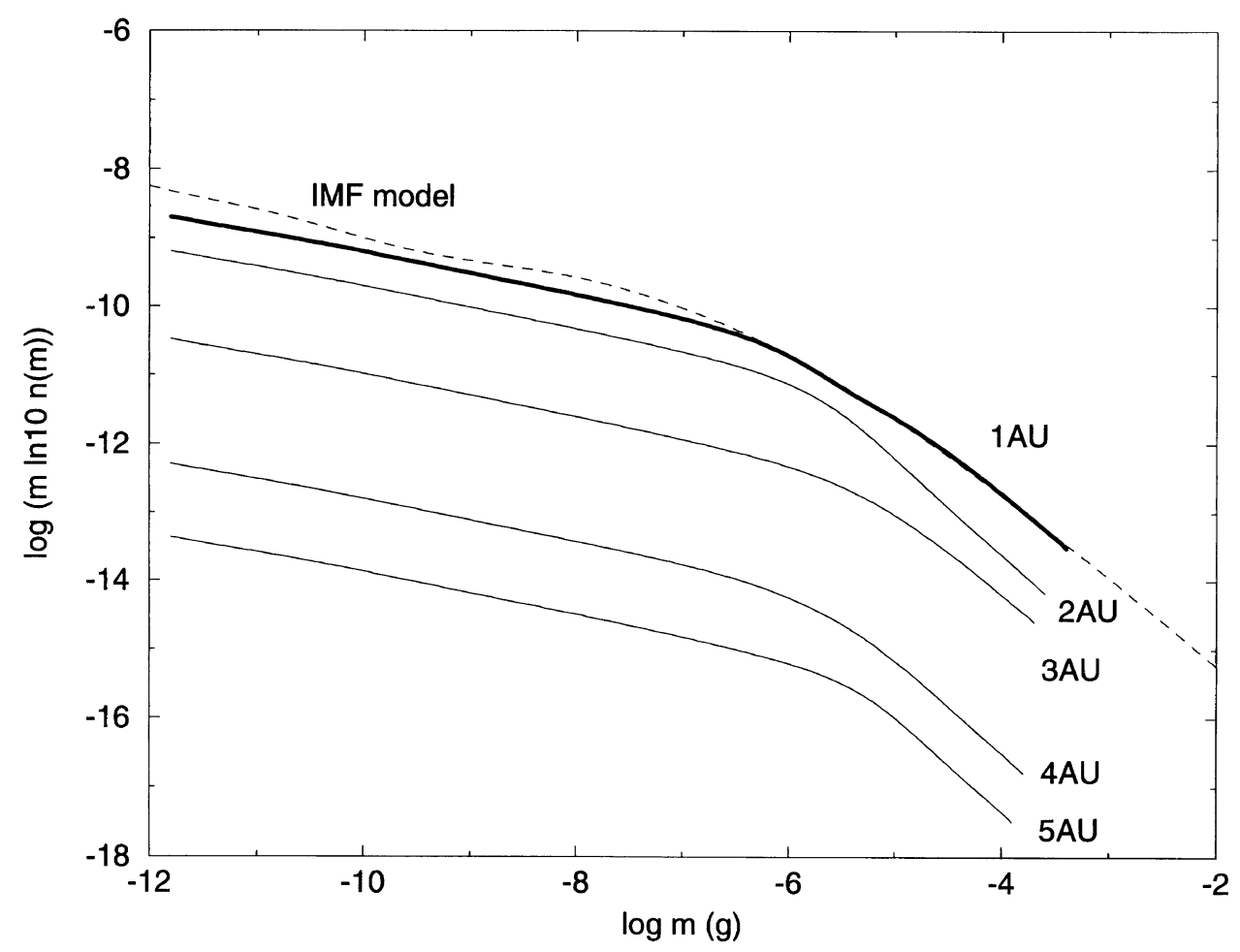

Fig. 4. Expected radial dependence of the number density distribution of interplanetary dust, from the particles by asteroids and comets. The loss terms are the same as Fig. 2, but the collisions of interstellar particles are applied in the parameter $C=0.1$.

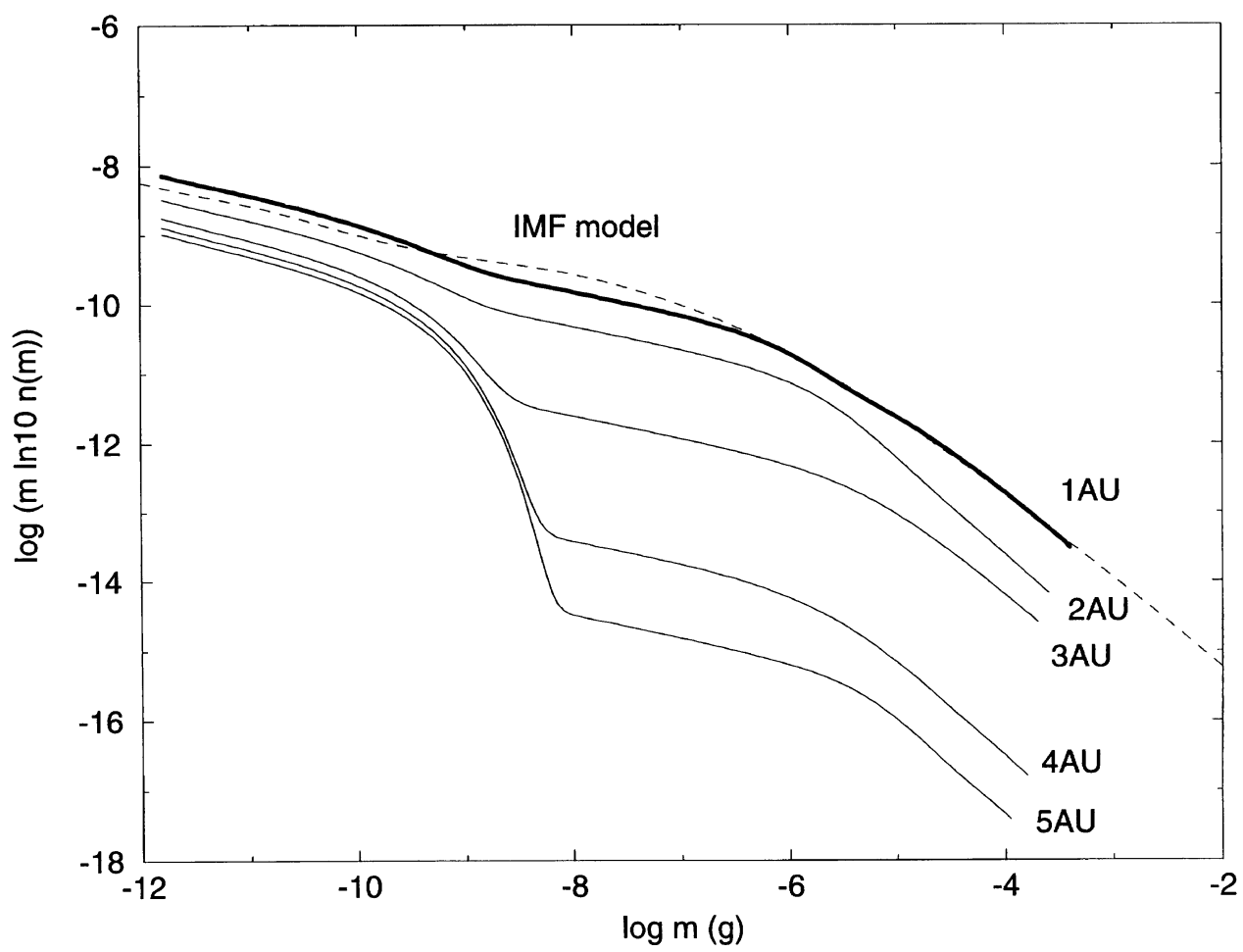

Fig. 5. The same as Fig. 4, but the influx of EKO dust is taken into account in the case of Fig. 4. The contribution of EKO dust in the interplanetary dust flux at $1 \mathrm{AU}$ is set to be comparable to that of IMF model in mass range $10^{-12} \mathrm{~g} \leq m \leq 10^{-9} \mathrm{~g}$. 


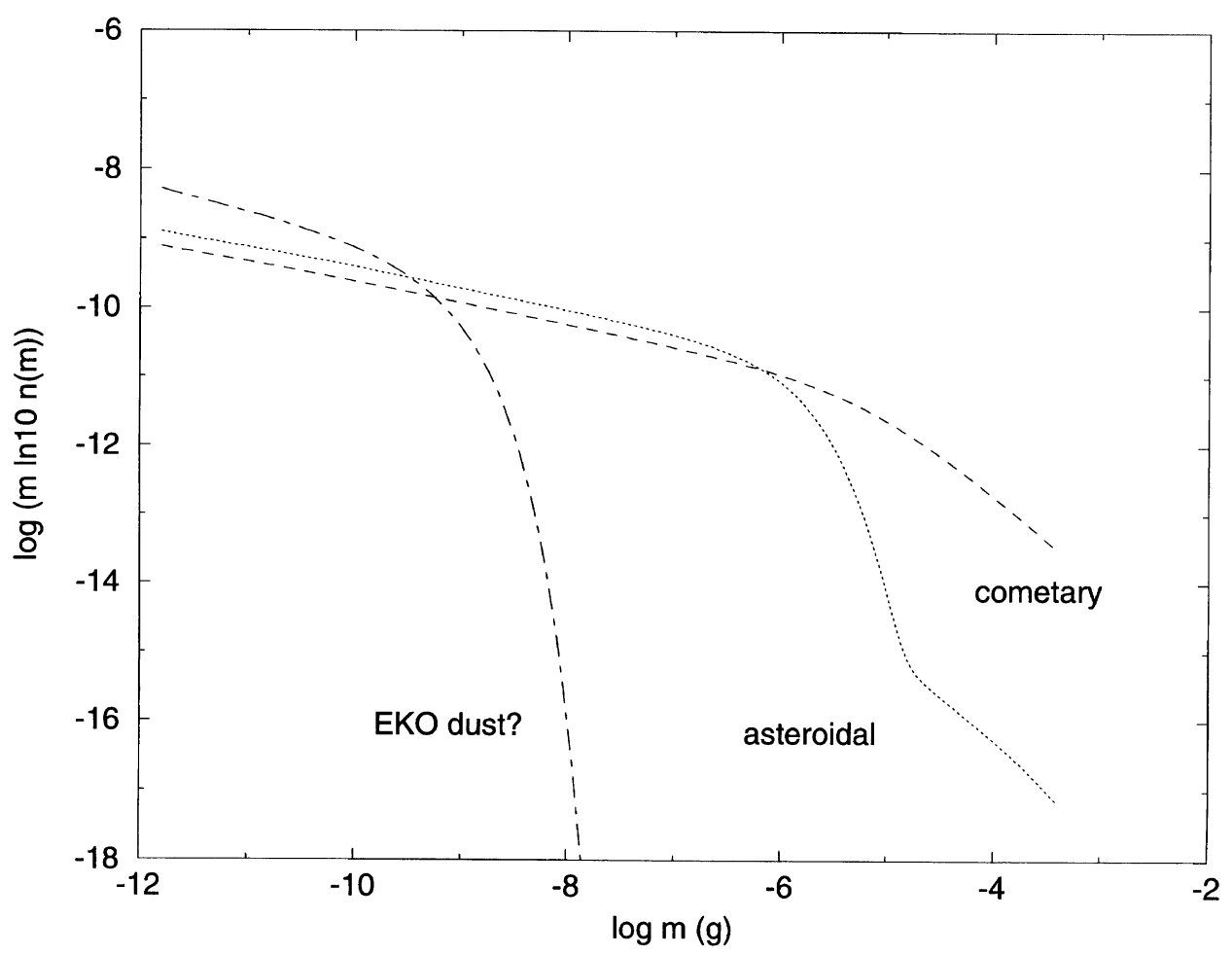

Fig. 6. Number density distribution for potential dust populations as a function of dust mass. The total number density distribution consisting of these three components is set to become the same as the result at $r=1$ AU shown in Fig. 5.

about three orders of magnitude at the outer edge of the asteroid belt, i.e. $4 \mathrm{AU}$, compared to that at $1 \mathrm{AU}$. According to the Galileo/Ulysses observations, the flux of dust particles in low-eccentricity low-inclination orbits seems to be reduced significantly outside $3 \mathrm{AU}$ (e.g. Baguhl et al., 1995b). This implies that the contribution of EKO particles shown in Fig. 5 is rather unlikely. However, we cannot rule out the existence of EKO particles since EKO can produce many ejecta particles in the EKO region (e.g. Backman et al., 1995; Stern, 1996) and about $20 \%$ of micron-sized particles can avoid gravitational scattering by outer planets in their orbital evolution (Liou et al., 1996) To be consistent with the Galileo/Ulysses results, the real contribution of EKO dust will be smaller than that in Fig. 5 .

Figure 6 shows the relative contribution of the individual dust groups to that detected at $1 \mathrm{AU}$, which is derived from the same calculations as those in Fig. 5. As noted before, the absolute values of the number density for each population have no strict meaning. This result only suggests that the asteroidal, cometary, and EKO dust may coexist at $1 \mathrm{AU}$ with comparable values of number densities of particles with masses of $10^{-12} \mathrm{~g} \leq m \leq 10^{-9} \mathrm{~g}$. For particles with masses of $10^{-9} \mathrm{~g} \leq m \leq 10^{-6} \mathrm{~g}$, EKO dust has no significant contribution, even if it exists. In the mass range of $m \geq 10^{-6} \mathrm{~g}$, the main group of such larger interplanetary dust has cometary origin.

The geometrical cross section $G$ of each dust group as a function of a solar distance is shown in Fig. 7, where $G=$ $\int_{m_{1}}^{m_{2}} \pi(3 m / 4 \pi \rho)^{2 / 3} n(m) d m$ and the mass density $\rho$ is set to $2.5 \mathrm{~g} \mathrm{~cm}^{-3}$. It is found that near $1 \mathrm{AU}$, the dominant group of the local geometrical cross section changes. Namely, the asteroidal dust group is dominant from $1 \mathrm{AU}$ to $3 \mathrm{AU}$ whereas inside $1 \mathrm{AU}$ the cometary dust group becomes the majority of the total cross section.

\subsection{Comparison with the Divine model}

It is interesting to compare the results of this work with Divine's five populations model (Divine, 1993). Needless to say, the method of deriving the dust groups described in this work is quite different from that of Divine's. In Divine's modelling, there are no direct explanations about relationships between dust populations and their origins. Furthermore, interactions such as mutual collisions between these dust populations are neglected. However, the five populations model has been constructed carefully, based on the data obtained by in-situ dust measurements and ground based observations. Therefore, Divine's results may have some worth, in particular for the orbital distribution of interplanetary particles. Since our discussions are limited to particles with masses $m \geq 10^{-12} \mathrm{~g}$, we cannot examine the "eccentric population" of small particles in the Divine model. Also, we are not unduly concerned about the "halo population", because this dust population was designed before interstellar dust flux was confirmed and should therefore be revised, particularly the typical orbital elements and mass range of the particles included in the "halo population".

The most critical difference between this work and the Divine model is the spatial and number density distribution of the "asteroidal population". Although the Divine model does not show explicitly that the "asteroidal population" is the dust population of asteroidal origin, it is reasonable to assume that the asteroidal particles are produced mainly in the asteroid belt. We found that particles with masses of $m \geq 10^{-6} \mathrm{~g}$ 


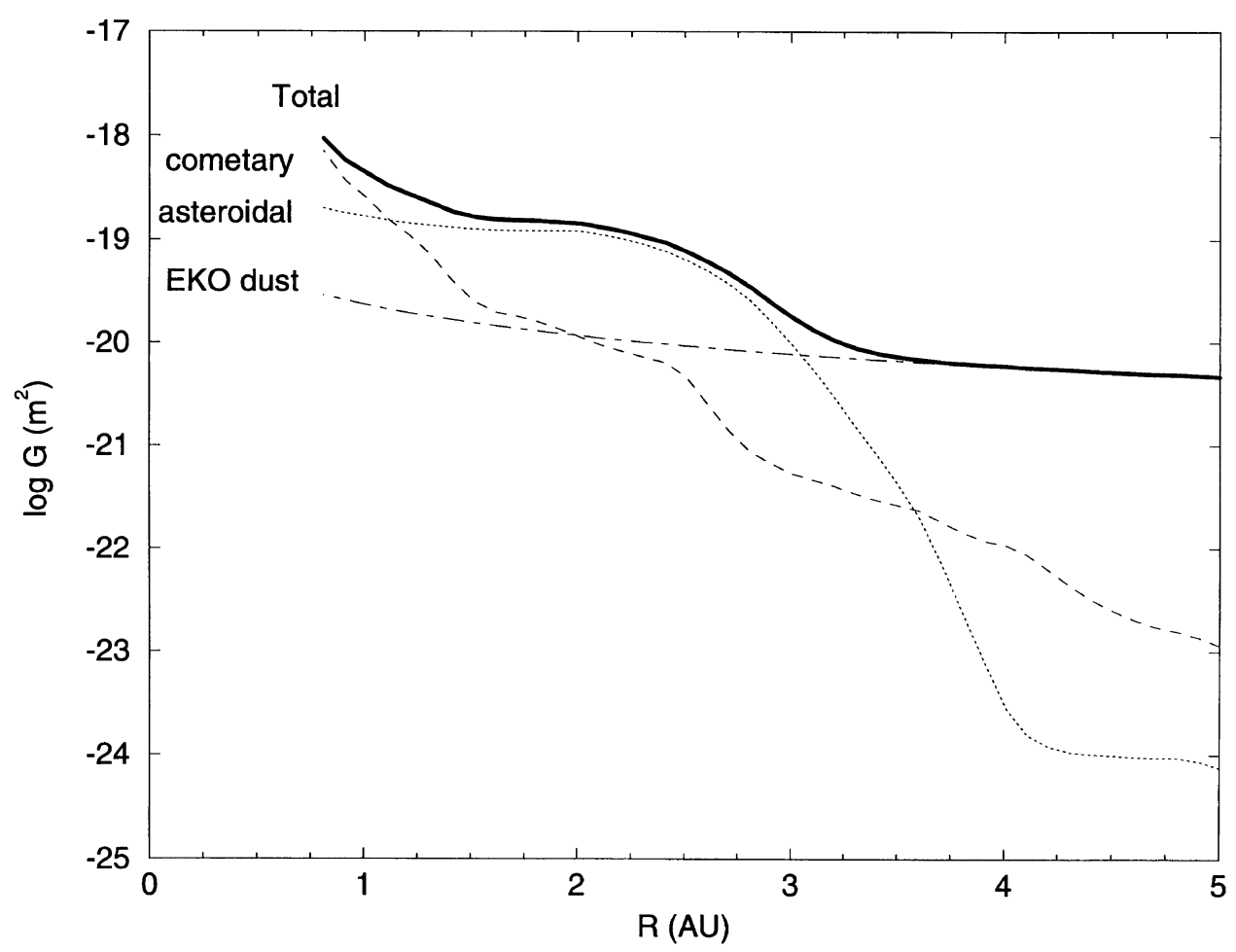

Fig. 7. Cross sectional distribution for the dust populations outside $1 \mathrm{AU}$ as a function of solar distance $R$.

generated in the asteroid belt could not arrive at $1 \mathrm{AU}$ without suffering mutual collisions. Therefore, the number density of asteroidal particles predicted at $1 \mathrm{AU}$ shows a rapid decrease beyond $m \geq 10^{-6} \mathrm{~g}$ as shown in Fig. 6. The position of Divine's "asteroidal population" at 1 AU may be converted by the cometary dust group used here. As mentioned before, the cometary particles are needed to explain the flux curve of the IMF in $m \geq 10^{-6} \mathrm{~g}$.

It is also reasonable to assume that the "core population" of the Divine model is a mixture of the particles of multiple origins in our model. In particular, the particles with $10^{-12} \mathrm{~g} \leq m \leq 10^{-9} \mathrm{~g}$ are not sensitive to their collisional loss, and consequently the coexistence of a significant amount of EKO dust in this mass range cannot be ruled out in the vicinity of $1 \mathrm{AU}$, as well as particles with asteroidal and cometary origins. It may be difficult to decide the relative abundance of each dust group in this mass range at $1 \mathrm{AU}$. However, the major contribution can be estimated from the radial dependence of the number density and cross sectional distribution (see Fig. 7).

We found that the contribution of asteroidal particles in the total geometrical cross section is almost comparable with that of cometary particles at $1 \mathrm{AU}$. However outside $1 \mathrm{AU}$, particles of asteroidal origin will predominate, whereas inside $1 \mathrm{AU}$ the cometary dust group will be prominent. This result is consistent with the existence of the "inclined population" in the Divine model inside $1 \mathrm{AU}$, because the average inclination of cometary particles is larger than that of asteroidal particles in general.

\section{Conclusions}

We have examined the number density distribution of interplanetary dust beyond $1 \mathrm{AU}$, taking into account the particles' orbital evolution, and including mutual collisions between particles. According to the IMF model, the dust flux curve in $m \geq 10^{-12} \mathrm{~g}$ observed at 1 AU changes its slope around $m=10^{-6} \mathrm{~g}$. We have found that this dependence of the dust flux is a result of the orbital and collisional evolution of particles ejected from their parent bodies. As the particles' mass increases, the timescale of the Poynting-Robertson decay increases, and as a result, the mutual collision of particles becomes important in the evolution of large particles. It is found that an effective dust supply originating directly from parent bodies near $1 \mathrm{AU}$ is necessary to explain the wide mass range of the flux curve at 1 AU. Furthermore, we have shown that short-period comets are good candidates for the parent bodies of the large particles at $1 \mathrm{AU}$.

We have designed an available model for the number density distribution of particles beyond 1 AU considering a significant contribution of asteroidal and EKO particles for $10^{-12} \mathrm{~g} \leq m \leq 10^{-9} \mathrm{~g}$. It is reasonable to assume that the interplanetary dust flux at $1 \mathrm{AU}$ consists of particles in multiple groups distinguished by their origins. Although we have not examined the absolute values of the number densities for asteroidal and EKO particles with $10^{-12} \mathrm{~g} \leq m \leq 10^{-9} \mathrm{~g}$, our results indicate the relative importance of each dust group. Namely, EKO particles can contribute to the $1 \mathrm{AU}$ flux only in the mass range $m \leq 10^{-9} \mathrm{~g}$ and asteroidal particles in the range $m \leq 10^{-6} \mathrm{~g}$, whereas cometary particles will contribute to a wide mass range of particles observed at $1 \mathrm{AU}$.

Furthermore, we have examined the radial dependence of the cross sectional distribution for each dust group. Since 
the radial distribution of the dust production rate depends on that of the parent bodies, the dominant dust group may change as the solar distance changes. We have shown that the asteroidal dust group becomes dominant between $1 \mathrm{AU}$ and $3 \mathrm{AU}$, while the cometary dust group will dominate inside 1 AU. However, it will not be sufficient to extrapolate the calculations described here to estimate the number density distribution inside $1 \mathrm{AU}$, because of our rough estimation of the dust production of comets, and the number and radial distribution of comets themselves.

Moreover, we potentially neglected the spatial inhomogeneity of cometary and asteroidal particles. Large dust particles ejected from a parent body maintain an orbit similar to that of their parent body until gravitational and collisional effects disperse them into the sporadic background. In particular, large cometary particles form cometary trails, and the dynamical evolution of trail particles, including the evolution of their size distribution, may play an important role in the time-averaged flux distribution at an arbitrary solar distance.

Mutual collisions between particles may produce many fragments inside $1 \mathrm{AU}$, which were neglected in this work. Such fragments may accelerate the collisional evolution of particles. Furthermore, some of the fragments produced inside $1 \mathrm{AU}$ will become an additional component of $\beta$ meteoroids. Unfortunately, the fragmentation of particleparticle collisions has been little studied. Further investigation and measurement will be needed to resolve the evolution of interplanetary dust particles in the entire mass range.

Acknowledgments. I would like to thank T. Mukai for helpful comments on several points in this paper. I am especially grateful for the reviews of E. Grün.

\section{References}

Backman, D. E., A. Dasgupta, and R. E. Stencel, Model of a Kuiper belt small grain population and resulting far-infrared emission, Astrophys. J., 450, L35-L38, 1995.

Baguhl, M., D. P. Hamilton, E. Grün, S. F. Dermott, H. Fechtig, M. S. Hanner, J. Kissel, B.-A. Lindblad, D. Linkert, G. Linkert, I. Mann, J. A. M. McDonnell, G. E. Morfill, C. Polanskey, R. Riemann, G. Schwehm, P. Staubach, and H. A. Zook, Dust measurements at high ecliptic latitudes, Science, 268, 1016-1019, 1995a.

Baguhl, M., E. Grün, D. P. Hamilton, G. Linkert, R. Riemann, P. Staubach, and H. A. Zook, The flux of interstellar dust observed by Ulysses and Galileo, Space Sci. Rev., 72, 471-476, 1995 b.

Dermott, S. F., P. D. Nicholson, J. A. Burns, and J. R. Houck, Origin of the solar system dust bands discovered by IRAS, Nature, 312, 505-509, 1984.

Dermott, S. F., D. D. Durda, B. Å. S. Gustafson, S. Jayaraman, J. C. Liou, and Y. L. Xu, Zodiacal dust bands, in Asteroids, Comets, Meteors 1993, edited by A. Milani et al., pp. 127-142, 1994a.

Dermott, S. F., S. Jayaraman, Y. L. Xu, B. Å. S. Gustafson, and J. C. Liou, A circumsolar ring of asteroidal dust in resonant lock with the Earth, Nature, 369, 719-723, 1994b.

Divine, N., Five populations of interplanetary meteoroids, J. Geophys. Res., 98, 17029-17048, 1993.
Draine, B. T. and H. M. Lee, Optical properties of interstellar graphite and silicate grains, Astron. J., 285, 89-108, 1984.

Grün, E., H. A. Zook, H. Fechtig, and R. H. Giese, Collisional balance of the meteoritic complex, Icarus, 62, 244-272, 1985.

Grün, E., H. A. Zook, M. Baguhl, A. Balogh, S. J. Bame, H. Fechtig, R. Forsyth, M. S. Hanner, M. Horanyi, J. Kissel, B.-A. Lindblad, D. Linkert, G. Linkert, I. Mann, J. A. M. McDonnell, G. E. Morfill, J. L. Phillips, C. Polanskey, G. Schwehm, N. Siddique, P. Staubach, J. Svestka, and A. Taylor, Discovery of jovian dust streams and interstellar grains by the Ulysses spacecraft, Nature, 362, 428-430, 1993.

Grün, E., M. Baguhl, N. Divine, H. Fechtig, D. P. Hamilton, M. S. Hanner, J. Kissel, B.-A. Lindblad, D. Linkert, G. Linkert, I. Mann, J. A. M. McDonnell, G. E. Morfill, C. Polanskey, R. Riemann, G. Schwehm, N. Siddique, P. Staubach, and H. A. Zook, Three years of Galileo dust data, Planet. Space Sci., 43, 953-969, 1995a.

Grün, E., M. Baguhl, N. Divine, H. Fechtig, D. P. Hamilton, M. S. Hanner, J. Kissel, B.-A. Lindblad, D. Linkert, G. Linkert, I. Mann, J. A. M. McDonnell, G. E. Morfill, C. Polanskey, R. Riemann, G. Schwehm, N. Siddique, P. Staubach, and H. A. Zook, Two years of Ulysses dust data, Planet. Space Sci., 43, 971-999, 1995b.

Grün, E., P. Staubach, M. Baguhl, D. P. Hamilton, H. A. Zook, S. Dermott, B. Å. S. Gustafson, H. Fechtig, J. Kissel, D. Linkert, G. Linkert, R. Srama M. S. Hanner, C. Polanskey, M. Hoanyi, B.-A. Lindblad, I. Mann, J. A. M. McDonnell, G. E. Morfill, and G. Schwehm, South-north and radial traverses through the interplanetary dust cloud, Icarus, 129, 270-288, 1997.

Ishimoto, H., Model calculation for particle mass distribution inside $1 \mathrm{AU}$, Planet. Space Sci., 1998 (in press).

Jayaraman, S. and S. F. Dermott, Estimating the asteroidal component of the zodiacal cloud using the Earth's resonant ring, in Physics, Chemistry, and Dynamics of Interplanetary Dust, edited by B. A. S. Gustafson and M. Hanner, ASP conference series, 104, pp. 155-158, 1996.

Kessler, D. J., Derivation of the collision probability between orbiting objects: the lifetime of Jupiter's outer moons, Icarus, 48, 39-48, 1981.

Le Sergeant d'Hendecourt, L. B. and P. L. Lamy, On the size distribution and physical properties of interplanetary dust grains, Icarus, 43, 350-372, 1980.

Liou, J.-C., H. A. Zook, and S. F. Dermott, Kuiper belt dust grains as a source of interplanetary dust particles, Icarus, 124, 429-440, 1996.

Mazets, E. P., R. L. Aptekar, S. V. Golenetskii, Yu. A. Guryan, A. V. Dyachkov, V. N. Ilyinskii, V. N. Panov, G. G. Petrov, A. V. Savvin, R. Z. Sagdeev, I. A. Sokolov, N. G. Khavenson, V. D. Shapiro, and V. I. Shevchenko, Comet Halley dust environment from SP-2 detector measurements, Nature, 321, 276-278, 1986.

McDonnell, J. A. M., Microparticle studies by space instrumentation, in Cosmic Dust, edited by J. A. M. McDonnell, pp. 337-426, Wiley, Chichester, 1978.

Mukai, T., Dust from the comets, in Highlights of Astronomy, 8, pp. 305312, 1989.

Nakamura, A. and A. Fujiwara, Velocity distribution of fragments formed in a simulated collisional disruption, Icarus, 92, 132-146, 1991.

Reach, W. T., B. A. Franz, J. L. Weiland, M. G. Hauser, T. N. Kelsall, E. L. Wright, G. Rawley, S. W. Stemwedel, and W. J. Spiesman, Observational confirmation of a circumsolar dust ring by the COBE satellite, Nature, 374, 521-523, 1995.

Stern, S. A., Signatures of collisions in the Kuiper disk, Astron. Astrophys., 310, 999-1010, 1996.

Yamamoto, S. and T. Mukai, Dust production by impacts of interstellar dust on Edgeworth-Kuiper Belt objects, Astron. Astrophys., 329, 785-791, 1998 .

H. Ishimoto (e-mail: isimoto@kobe-u.ac.jp) 